\title{
Results and Analysis from Space Suit Joint Torque Testing
}

\author{
Jennifer Matty $^{1}$ \\ Jacobs Engineering and Science Contract Group (ESCG), NASA - Johnson Space Center, Houston, TX, 77058
}

\begin{abstract}
A space suit's mobility is critical to an astronaut's ability to perform work efficiently. As mobility increases, the astronaut can perform tasks for longer durations with less fatigue. Mobility can be broken down into two parts: range of motion (ROM) and torque. These two measurements describe how the suit moves and how much force it takes to move. Two methods were chosen to define mobility requirements for the Constellation Space Suit Element (CSSE). One method focuses on range of motion and the second method centers on joint torque. A joint torque test was conducted to determine a baseline for current advanced space suit joint torques. This test utilized the following space suits: Extravehicular Mobility Unit (EMU), Advanced Crew Escape Suit (ACES), I-Suit, D-Suit, Enhanced Mobility (EM)ACES, and Mark III (MK-III). Data was collected data from 16 different joint movements of each suit. The results were then reviewed and CSSE joint torque requirement values were selected. The focus of this paper is to discuss trends observed during data analysis.
\end{abstract}

\section{Nomenclature}

$\begin{array}{ll}\text { ACES } & =\text { Advanced Crew Escape Suit } \\ C S S E & =\text { Constellation Space Suit Element } \\ D A C & =\text { Data Access Components } \\ E M & =\text { Enhanced Mobility (ACES) } \\ E M U & =\text { Extravehicular Mobility Unit } \\ E S C G & =\text { Engineering and Science Contract Group } \\ I V A & =\text { intravehicular activity } \\ \text { LEA } & =\text { launch, entry, abort } \\ M K-I I I & =\text { Mark III } \\ \text { NASA } & =\text { National Aeronautics and Space Administration } \\ \text { psid } & =\text { pounds per square inch } \\ \text { ROM } & =\text { range of motion } \\ \text { SEAS } & \text { Science, Engineering, and Analysis Services } \\ \text { TMG } & =\text { thermal micrometeoroid garment }\end{array}$

\section{Introduction}

I

$\mathrm{n}$ an effort the create mobility requirements for Constellation Space Suit Element (CSSE), mobility was broken into two parts: range of motion (ROM) and torque requirements. Recently, testing was completed to characterize existing space suits' joint torque. The purpose of this test was to collect joint torque data on various suits in an effort to compare data, which could provide insight for selecting appropriate requirement values. This paper summarizes the test and discusses general data traits. While the test data was used for requirements development, the entire data set and requirement selection is not covered in the scope of this paper; however, they may be found in the Space Suit Joint Torque Test Report ${ }^{1}$.

\section{Test Summary}

To quickly benchmark several different suits' joint torque profiles, it was decided to pursue an unmanned testing approach using a modified fish scale method. This method uses a load cell to measure the force required to pull an unmanned space suit joint though its ROM. Some of the difficulties previously faced, such as removing gravity and human subject influence, were alleviated by removing such factors from the test design. The unmanned joint torque

\footnotetext{
${ }^{1}$ Space Suit Engineer, SEAS, 2222 Bay Area Blvd Houston, TX, AIAA Member .
} 
test utilized the Extravehicular Mobility Unit (EMU), Advanced Crew Escape Suit (ACES), I-Suit, D-Suit, Enhanced Mobility (EM)-ACES, and Mark III (MK-III) to investigate the effects of torque on each suit joint. All suits were tested without a thermal micrometeoroid garment (TMG) or cover layers, with the exception of the ACES, because the cover layer is also used as the restraint layer in some areas. Two pressures were used during the test: a nominal suit pressure [3.5, 3.75, and 4.3 pounds per square inch (psid)] and a representative Constellation Program vent pressure ( 0.8 psid).

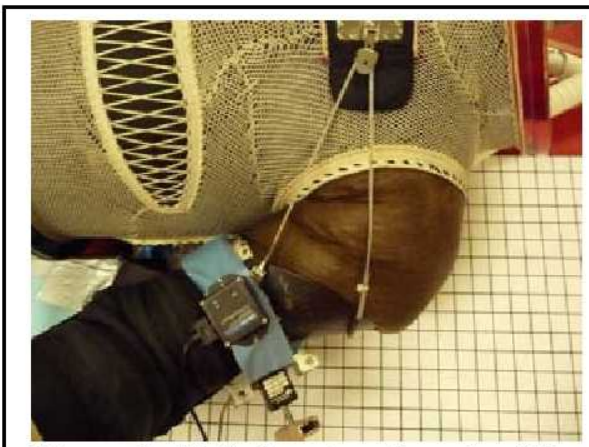

Figure 1. D-Suit setup for shoulder adduction /abduction. Data collection sensors attached to upper arm.

Preliminary testing determined that a miniature load cell, rated at 100 pounds, would be the best instrument to assess the force component, and a three-axis accelerometer would be used to measure the angle to derive the joint torque. A Data Access Components (DAC) system and custom LabVIEW user interface was created to capture the data. A test stand was necessary to support and restrain portions of the suit during the test. Attention was paid to setting up the desired joint movement in a gravity-neutral state. It was determined that no specialty test stands were required; a sturdy lab table or suit-specific donning stand was used in combination with ratchet straps and foam blocks for securing the suit during testing. A grid board was used to aid in tracking the ROM in video and, if necessary, to support the appendage or guide the movement in a single plane. The grid board was held in place by bar clamps and a tripod-style c-stand used in the motion picture industry. An overhead video system was used to record video during testing. Several custom accessories were made to complete the test set up. A bracket and handle were fabricated to mount the load cell onto the appendage (see Fig. 1). Various products such as hook and loop strips, Coban ${ }^{\mathrm{TM}}$ tape, screws, binder clips, and residue-free tape were used to secure parts of the suit or to mount the data collection equipment. During testing, some

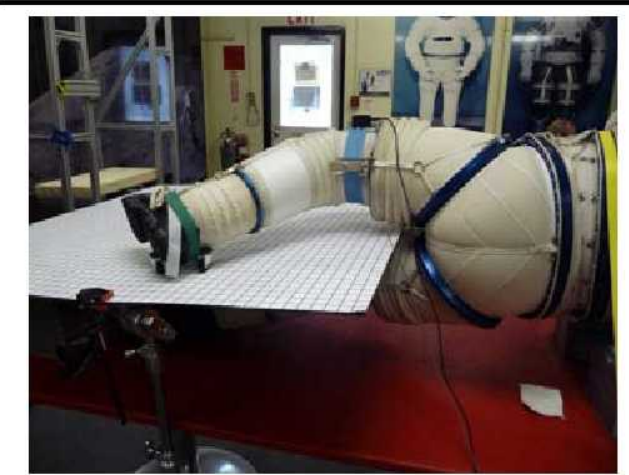

Figure 2. I-Suit set up for hip adduction/abduction. I-suit utilized tricaster plant holder to support boot. problems arose regarding friction caused by heavy suit parts, such as wrist bearings and boots, dragging along the grid board. To alleviate this problem, a series of transfer balls and casters were reviewed, along with brackets to attach them; however, the best solution was the use of a tri-caster plant holder (see Fig. 2).

Each test began with securing the pressurized, unmanned suit to a restraint table using ratchet straps or a donning stand (see Fig. 3). Special attention was paid to this step; it was critical that during each test, only the joint tested could be allowed to move. If the table or portions of the suit not being tested moved during a trial, it could impact the data being collected, adding additional variance to the angle or torque data. For each joint movement the suit was

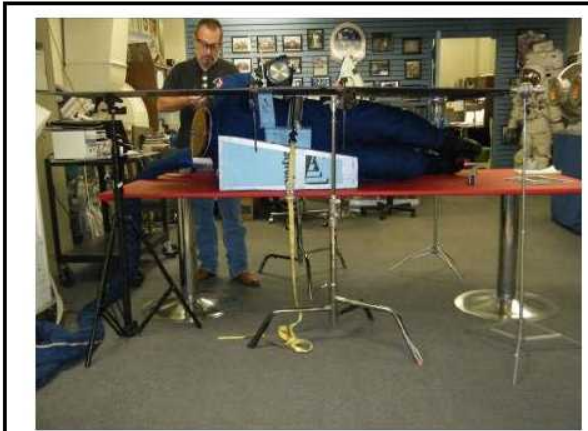

Figure 3. EM-ACES set up for shoulder Flexion /Extension. Suit restrained with ratchet straps and supported with foam. configured such that the selected joint was parallel to the floor, thus reducing the effects of gravity. Foam blocks were used to support the suit and to help align the joints correctly. The data collection devices were then attached to the joint. Generally, the accelerometer was attached to the top of the rotating segment of the joint being moved. The load cell was required to be perpendicular to the joint axis and parallel to the floor. It was also equally important for data collection devices to be adequately secured to the suit. Next, the selected joint was pulled by hand through its ROM at each pressure. All tests were pulled by the same individual. This test was repeated for each pressure, suit, and joint. The following joint movements were collected if mobility features were present on the suit tested: shoulder flexion/extension, shoulder adduction/abduction, shoulder medial/lateral, shoulder extension/internal rotation, elbow flexion/extension, torso rotation, torso flexion/extension, hip flexion/extension, hip adduction/abduction, hip extension/internal 
rotation, knee flexion/extension, ankle flexion/extension, ankle extension/internal rotation, wrist flexion/extension, wrist adduction/abduction, wrist pronation/supination.

Data was collected throughout the test by the accelerometer and load cell. Video data was collected overhead and still photographs were taken at various intervals and locations. Raw force and position data was processed by a program using LabVIEW and exported to Microsoft Office Excel ${ }^{\circledR}$. The force data in the Excel ${ }^{\circledR}$ spreadsheet was then converted to torque by adding an appropriate distance multiplier. The distance multiplier was determined by measuring the distance from the position of the load cell to the point of rotation. If the joint had multiple points of rotation, such as with a multi-bearing joint, the distance used was the approximation of where the human joint would be located. This allows for a complex joint mobility system's torque to be treated as a single simplified action. The position data was then reoriented to align with the ROM reference figures to produce the angle data. The reorientation was necessary to standardize the data and to make direct comparisons to the existing ROM requirements data.

\section{Test Points of Interest}

This joint torque test is the most comprehensive of its kind, comparing 6 different space suits and 16 major joints. While a complete report has been compiled for all the joints at various pressures, a selection of the results has been chosen to demonstrate the various joint traits that can be identified in the charts. Seven chart types were selected for further discussion.

\section{A. Basic Single Axis}

The most basic chart is a single axis joint such as a wrist or ankle. The wrist adduction/abduction chart shown below (see Fig. 4) demonstrates a hysteresis pattern with equal peak torques on at each of the ROM extremes. The Phase VI glove uses a gimbal system to provide stability during the adduction/abduction movement. This allows the joints to remain steady and equal for both sides of the neutral position. Other joints perform in a similar manner, but may have variations depending on the design. For example, a knee or elbow joint follows a similar pattern; however, such joints are only required to move from a neutral or fully extended position to a flexed position on one side. As a result, some designs are not bidirectional. This results in a chart that appears cut in half, the data trends to zero torque at their neutral position, but exhibit a hysteresis curve as they approach there maximum flexed angle.

\section{Phase VI 4.3 psid Wrist Adduction/Abduction}

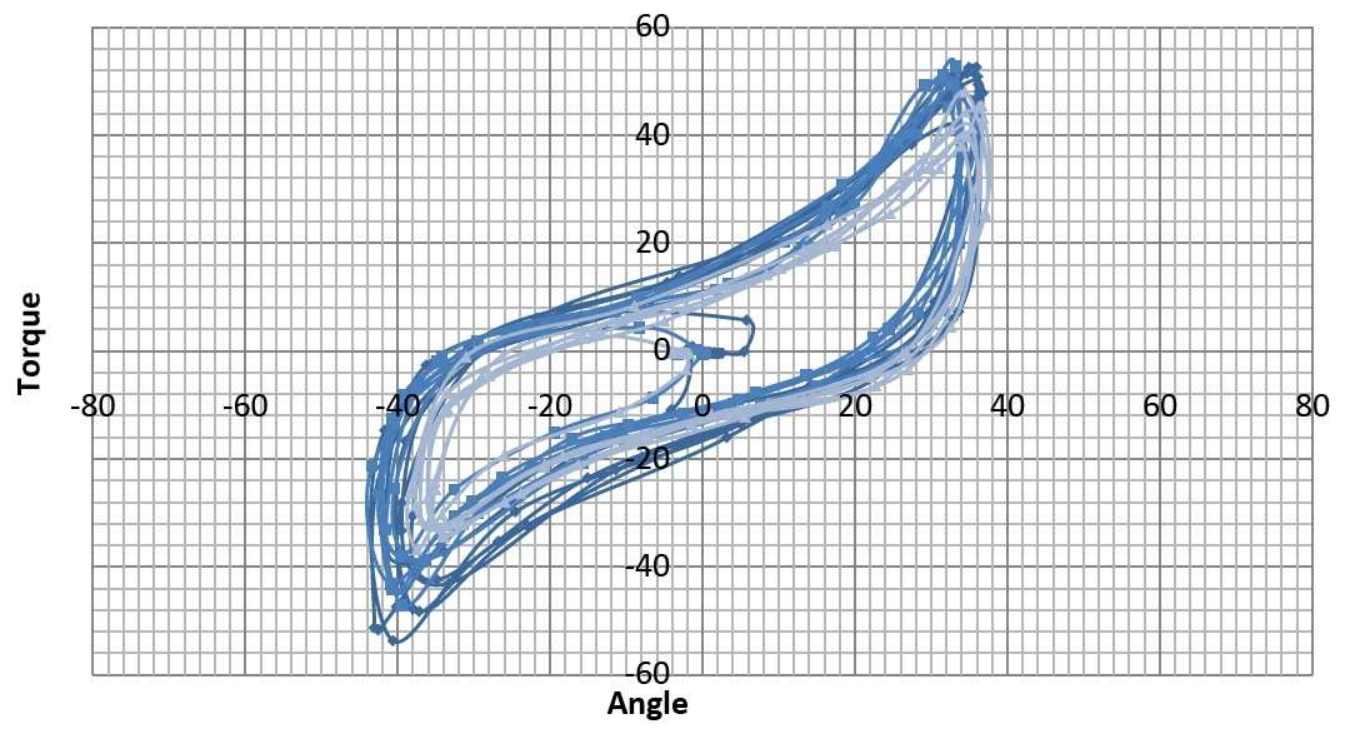

Figure 4. Torque profile for single axis joint. Phase VI glove at 4.3 psid during wrist adduction/abduction. 
The basic single axis joint example applies to nearly all non-bearing joints. As the ROM changes, the torque magnitudes change the shape with changes, but still take the same general shape with smooth transitions between each ROM extreme.

\section{B. Bearings}

The second type of chart is of a single bearing. All suits used in this test had a minimum of one bearing used to gain additional low torque ROM. The most common and simple bearing is the one used in each suits' wrist. Nothing compares to the mobility gains from a well-placed bearing; however, tradeoffs such as weight, cost, safety, and complexity prevent their widespread use, especially in suits used for launch, entry, abort (LEA) or intravehicular activity (IVA). The bearing joint torque profile is simple. Since bearing joints have unlimited ROM, they encompass a full 360 degrees of rotation and should exhibit identical torques when either pushed or pulled through their ROM. In the example bearing chart below (see Fig. 5), the EM-ACES scye bearing demonstrated the expected profile of a bearing. This chart shows the bearing had little variance as it moved through its ROM. It also shows the trademark turn point where the top portion connected to the bottom. In Fig. 5, this occurred at approximately - 100 degrees. At this point, there was also a slight increase in torque as the bearing reversed, showing evidence of a breaking torque. All bearing charts resemble this example as long as the bearing is completely parallel with the floor and other suit artifacts do not impede the path of the bearing.

\section{EM-ACES 4.3 psid Shoulder Flexion/Extension}

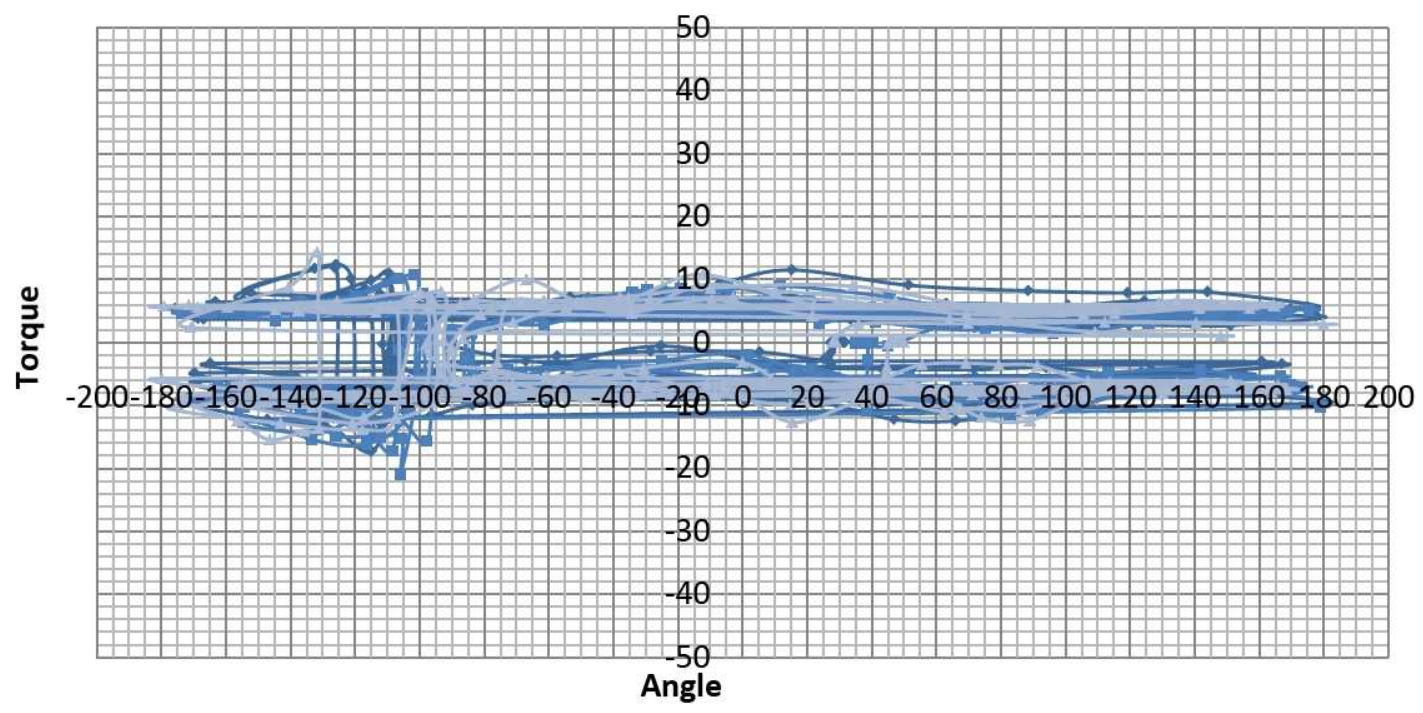

Figure 5. Torque profile for bearing joint. EM-ACES shoulder at 4.3 psid during flexion/extension.

The basic bearing joint example applies to joints where a single bearing is in line with the joint rotation. If the bearing and joint movement are not in line or if they are not parallel to the floor, the data may take the shape of an arch.

\section{Strong Neutral}

The third trait that characterizes a strong neutral point in some joints is a strong preference for a joint to bounce back to its original starting position. In the chart below (see Fig. 6), the I-Suit ankle joint demonstrates this trait well. In this chart, the data appears to be similar to the basic single axis charts; however, the data exhibits a "waist" at the neutral point. This effect is especially prevalent in joints that have little mobility designed into them, and such effect can have relatively high torque profiles when moved away from the neutral point of the suit. The suit's individual design can dictate a neutral point that has little to do with the neutral point or origin for the ROM collected. A suit like the ACES can have a hip that is designed such that it takes on a sitting position when pressurized. This strong bias towards a flexed hip with no features to aid in mobility will demonstrate this effect. 


\section{I-Suit 4.3 psid Ankle Dorsi/Plantar}

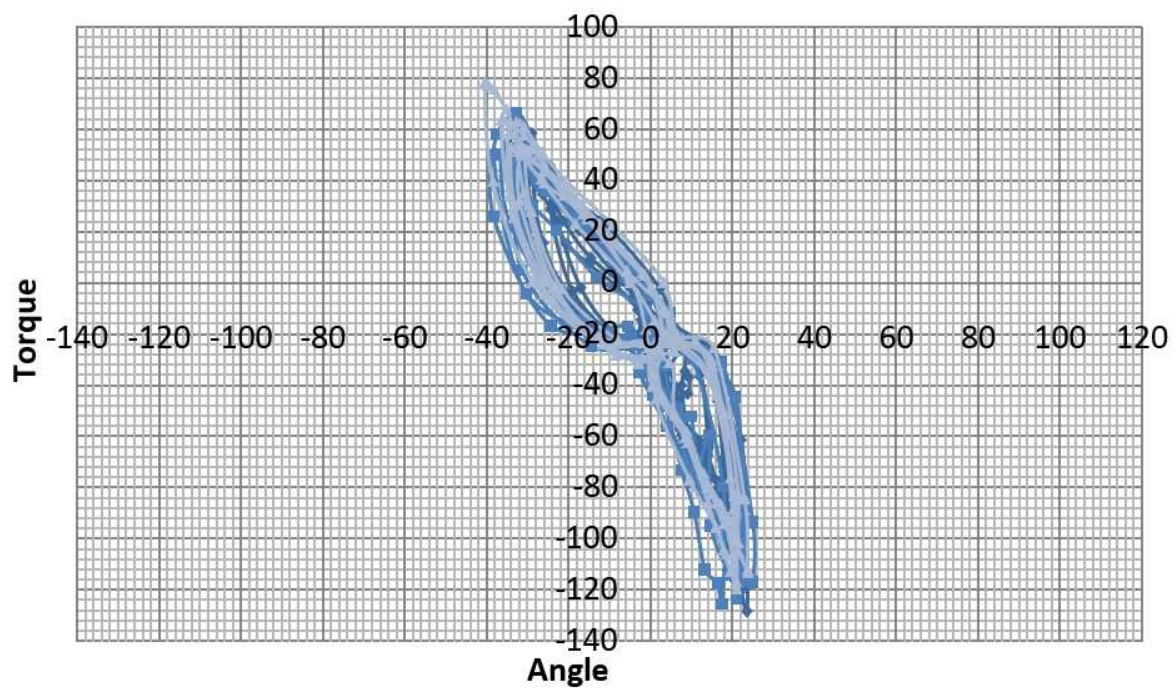

Figure 6. Torque profile for a strong neutral joint. I-Suit ankle at 4.3 psid during dorsi/plantar flexion.

A suit with ample mobility features can also exhibit this trait. The example shown in Fig. 6 is a good representative. The I-Suit ankle flexion joint has several convolutes to aid in mobility; however, it still has a strong preference to spring back to its neutral point.

\section{Two Phase}

The traits of a two-phased joint can be seen in the profile of the D-Suit shoulder's adduction/abduction chart (see Fig. 7). A two-phased joint is identified by two independent actions of the joint or breaks in the torque profile. For the D-Suit's shoulder example, the chart identifies a large increase in torque to move the joint from -10 to -40 degrees. This is followed by a rapid decrease at -80 degrees and a section of fairly uniform torque with a slight increase around -120 degrees where the joints' softgoods are being overdriven. 


\section{D-Suit 3.75 psid Shoulder Adduction/Abduction}

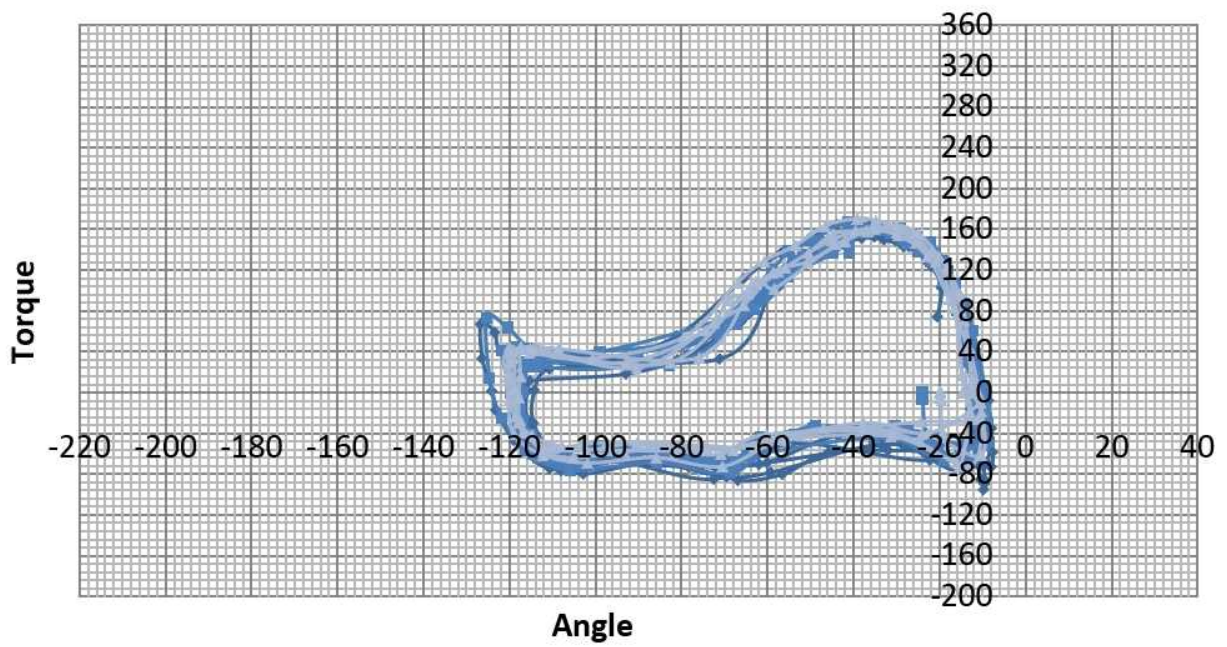

Figure 7. Torque profile for a two-phase joint. D-Suit shoulder at 3.75 psid during adduction/abduction.

\section{E. Winged Bearing Flip}

Another type of two phased joint can be described as a winged bearing flip. This trait can be identified by the winged shape the data plots appear to take. (see Figure 8) This shape indicates that the joint was pulled in one direction from the neutral point then also pulled in the opposite direction. It appears that the joint takes on 2 halves of a basic single axis joint where the half where it is pushed is cutoff since it does not have negative torque values.

\section{EM-ACES 4.3 psid Shoulder Interior/Exterior}

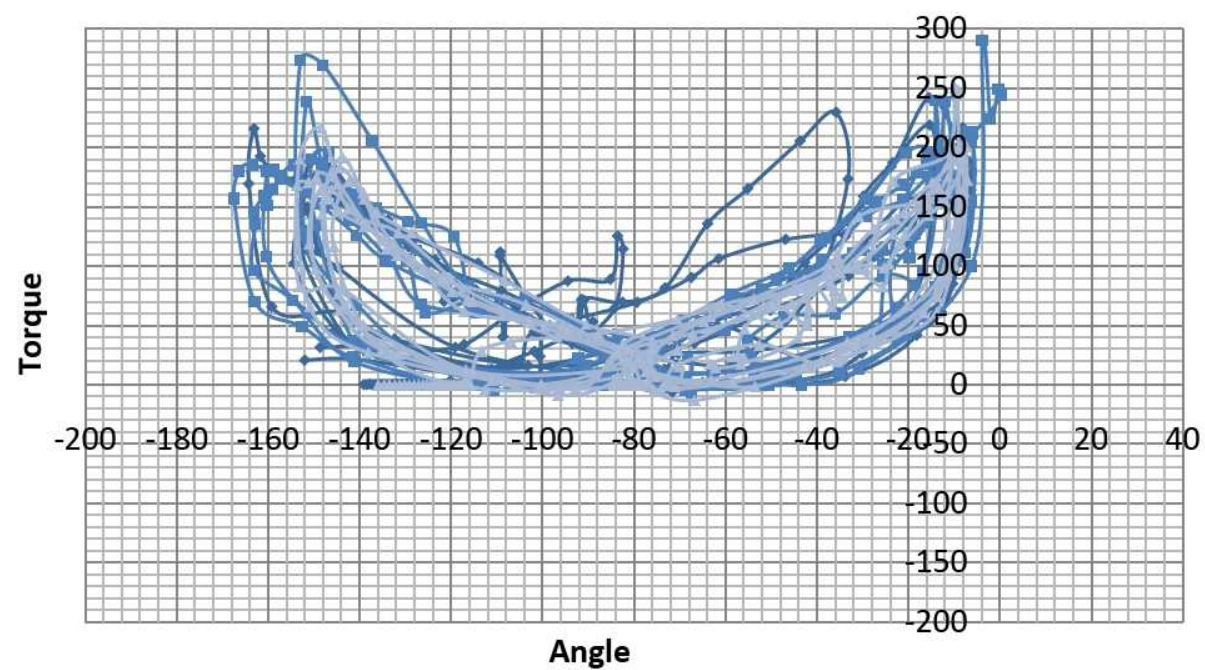

Figure 8. Torque profile for a winged bearing flip joint. EM-ACES shoulder at 4.3 psid during interior/exterior transverse rotation. 
Shoulders with a scye bearing demonstrated this trait; the way this test was performed the data collection sensors were located at the upper arm bearing. The joint was first pulled toward the front of the suit to reach a maximum interior angle, and then it was pushed to gain a maximum exterior angle. However, after it reached the neutral point, the scye bearing engaged and flipped the bearing such that the upper arm segment was once again pulled to its maximum angle (this time to its exterior). This movement is what cause the winged pattern in the data.

\section{F. Butterfly}

A similar pattern, described as a butterfly pattern, was produced by two slightly different data collection locations. There was difficulty collecting data at low pressures from joints that have large volumes such as the upper leg segment. As the pressure decreased, the large segment was easy to deform if the load was not adequately distributed. During the hip flexion/extension test at $0.8 \mathrm{psid}$, all soft suits experienced this deformation when the load cell apparatus was pushed in towards the suit. However, pulling the segment in the same location resulted in minimal deformation. The solution to obtaining the complete ROM was to collect data from one side of the segment and then, without changing the orientation of the accelerometer, set the load cell on the opposite side of the segment to collect additional data. An example of this complete data collection can be seen in Fig. 9.

\section{EM-ACES 0.8 psid Hip Flexion/Extension}

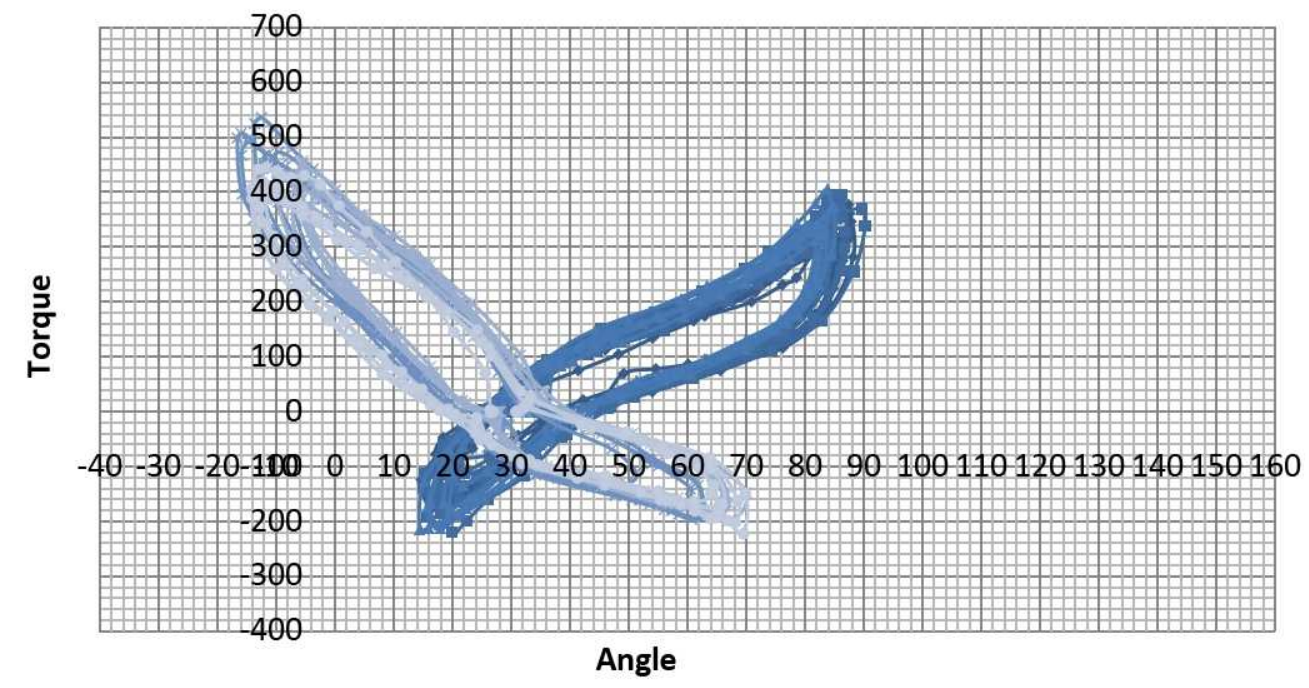

Figure 9. Torque profile for a butterfly joint. EM-ACES hip at 0.8 psid during flexion/extension.

A butterfly pattern could be observed for any basic single axis joint for data collected on both sides of a segment. However, it was only necessary to collect data from both sides if the segment deformed so much that the full ROM could not be collected from one side. If lower pressures were required, more joints would need to use this protocol. As the pressure was lowered, however, significant deformation started to be seen on the opposite side of the segment where the load was taken from the pull.

\section{G. Multi-Phase Sweep}

The most complicated data profile can be described as a multi-phase sweep. This profile was typically observed in joints where several mobility features came into play. It is just one step beyond a two-phase joint. The I-Suit hip adduction/abduction illustrates this data profile in the example chart shown in Fig. 10. The I-Suit uses a combination of mobility features to obtain hip adduction/abduction; a set of convolutes placed mid thigh and a hip bearing in the brief area. For this particular suit, two sets of data were collected. The first set only collected data for the lower set of convolutes; this appears darker in the chart and all data resides between 20 and -20 degrees. The second set collected used the full capability of both mobility features; this data produced a section similar to a basic single axis joint. It then transitioned to a section that characterized a bearing that is not completely in line with the joint rotation. 


\section{I-Suit 4.3 psid Hip Adduction Abduction}

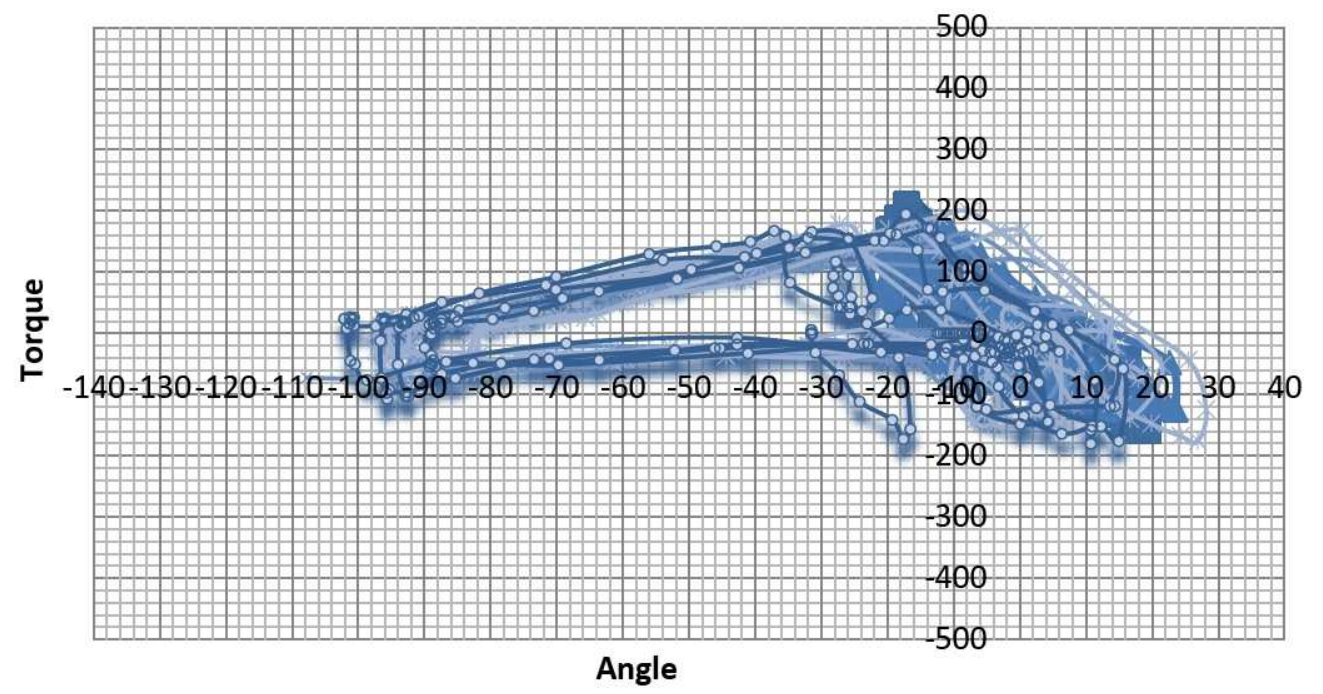

Figure 10. Torque profile for a multi phase sweep joint. I-Suit hip at 4.3 psid.

The I-Suit uses a combination of mobility features to obtain hip adduction/abduction, set of convolutes placed mid thigh and a hip bearing in the brief area. For this particular suit two sets of data were collected. The first set only collected data for the lower set of convolutes in the chart this appears darker and all the data resides between 20 and -20 degrees. The second set collected utilized the full capability of both mobility features; this data produced a section similar to a basic single axis joint it then transitions to a section that characterizes a bearing that is not completely in line with the joint rotation. A multi-phase sweep pattern would be observed anywhere there are multiple mobility features contributing to a joint movement.

\section{Conclusion}

While it was known at the beginning of the project that the data could provide only minimal insight to the level of exertion experienced by a crewmember, the test indicated that a certain torque requirement was met. The main goal was to identify an appropriate joint torque requirement for each of the major joints. This test provided ample information from the data collected to make these requirements. Moreover, the test also raised team confidence that a test performed in the same manner could be used to verify requirements for a new suit. Additional work should be completed to further understand the relationships between human and suit capabilities.

\section{References}

${ }^{1}$ Matty, J., “Space Suit Joint Torque Test Report,” NASA CTSD-CX-5304, 2010. 